\title{
Improved Confinement due to Open Ergodic Field Lines Imposed by the Dynamic Ergodic Divertor in TEXTOR
}

K. H. Finken, ${ }^{1}$ S. S. Abdullaev, ${ }^{1}$ M. W. Jakubowski, ${ }^{1}$ M. F. M. de Bock, ${ }^{2}$ S. Bozhenkov, ${ }^{1}$ C. Busch, ${ }^{1}$ M. von Hellermann, ${ }^{2}$ R. Jaspers, ${ }^{2}$ Y. Kikuchi, ${ }^{3}$ A. Krämer-Flecken, ${ }^{1}$ M. Lehnen, ${ }^{1}$ D. Schega ${ }^{1}$ O. Schmitz, ${ }^{1}$ K. H. Spatschek,${ }^{1,4}$ B. Unterberg, ${ }^{1}$ A. Wingen, ${ }^{4}$ R. C. Wolf, ${ }^{1}$ O. Zimmermann, ${ }^{1}$ and the TEXTOR team

${ }^{1}$ Trilateral Euregio Cluster, Institut für Plasmaphysik, Forschungszentrum Jülich, EURATOM Association, D-52425 Jülich, Germany ${ }^{2}$ FOM Institute for Plasma Physics Rijnhuizen, EURATOM Association, Postbus 1207, NL-3430 BE Nieuwegein, The Netherlands ${ }^{3}$ Department of Electrical Engineering and Computer Sciences, Graduate School of Engineering, University of Hyogo, 2167, Shosha, Himeji, 671-2280 Hyogo, Japan

${ }^{4}$ Institut für Theoretische Physik, Heinrich-Heine Universität Düsseldorf, D-40225 Düsseldorf, Germany

(Received 23 August 2006; published 5 February 2007)

The ergodization of the magnetic field lines imposed by the dynamic ergodic diverter (DED) in TEXTOR can lead both to confinement improvement and to confinement deterioration. The cases of substantial improvement are in resonant ways related to particular conditions in which magnetic flux tubes starting at the $X$ points of induced islands are connected with the wall. This opening process is connected with a characteristic modification of the heat deposition pattern at the divertor target plate and leads to a substantial increase and steepening of the core plasma density and pressure. The improvement is tentatively attributed to a modification of the electric potential in the plasma carried by the open field lines. The confinement improvement bases on a spontaneous density built up due to the application of the DED and is primarily a particle confinement improvement.

PACS numbers: 52.55.Fa, 52.30.-q, 52.35.Vd, 52.55.Tn

Helical magnetic field perturbations are introduced in tokamak plasmas in order to study the ergodic divertor concept [1-3], for confinement, improvement [4], and the interaction of such perturbations with the magnetohydrodynamic (MHD) stability of the plasma [5,6]. Recent experiments suggest ergodization as a control method to mitigate edge localized modes while maintaining the pedestal pressure and the plasma confinement [7]. The TEXTOR tokamak has been equipped with the dynamic ergodic diverter (DED) $[8,9]$ in order to study the interaction between helical magnetic field perturbations and plasma transport and stability.

The DED consists of 16 magnetic perturbation coils (four quadruples), plus two additional coils for the compensation of the magnetic field imperfections at the feeder regions of the coils. The coils wind helically around the inner side of the torus (major radius: $R=1.75 \mathrm{~m}$, minor radius of the circular plasma cross section typically $a=$ $0.47 \mathrm{~m}$ ) with a pitch corresponding to the magnetic field lines of the magnetic flux surface with a safety factor of $q=3$. Depending on the choice of coil connections to the power supplies, base modes with different poloidal and toroidal mode numbers can be produced. For the DED these are $m / n=12 / 4,6 / 2$, and 3/1. The penetration depth into the plasma strongly depends on the mode numbers: while the $m / n=12 / 4$ affects the edge plasma only, the $m / n=3 / 1$ mode reaches into the plasma center.

In this Letter we present a new experimental finding of an improved confinement scenario during the DED operation in the $m / n=6 / 2$ base mode; for the investigations presented here, the DED current was dc. The perturbation field of the DED coils generates the poloidal mode spectrum which contains the dominant sidebands with $3 \leq$ $m \leq 9$ centered at $m \approx 5 \div 6$ and the toroidal mode number $n=2$. The time traces of the plasma parameters in this improved confinement scenario is shown in Fig. 1. Displayed from top to bottom are the density, the plasma current, the DED current, and neutral beam heating power. The conditions for the target plasma are achieved at $1.8 \mathrm{~s}$. During the interval $1.8 \leq t \leq 3.3 \mathrm{~s}$ the plasma current is ramped down, keeping $B_{t}$, neutral beam power, and DED current constant. During the ramp-down phase one observes several maxima and minima in the electron density.

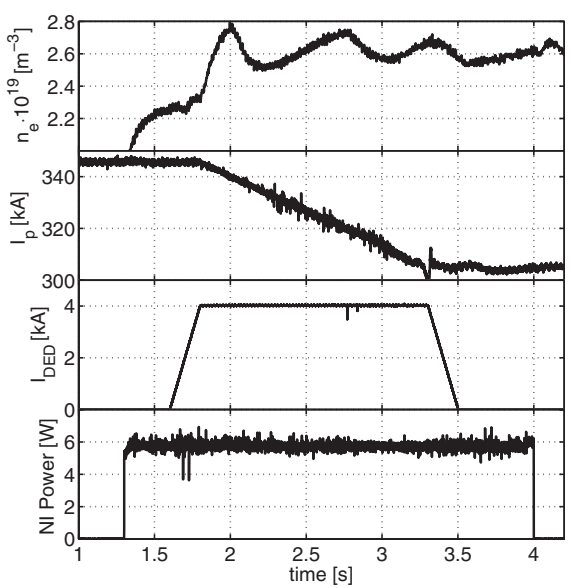

FIG. 1. Time traces of density, plasma current, DED current, and NBI heating power. Please note that the zero points of the density and plasma current are suppressed. 
Note that the zero of the density is suppressed in order to visualize better the density variation. The plasma current variation is equivalent to a variation of the safety factor $q(a)$ and it is well known that the degree of ergodization depends strongly on the safety factor [10,11].

The maxima in the density in Fig. 1 base on a sufficiently deep ergodization and the formation of magnetic flux tubes connecting the ergodic zone with the wall; the first maximum in the density trace of Fig. 1 corresponds to a penetration of the DED field the $q=5 / 2$ island chain and the second maximum to the $q=9 / 4$ island chain. The deepest penetration without provoking disruptions reaches up to $q=2$. In Fig. 2(a) a scenario is shown which proved to be the case with strongest improvement when the DED is applied; shown are the density, the DED current, and a time scan of the poloidal target temperature profile (line scans). In this example, the plasma current is fixed to the optimum value of the current scan $\left(I_{p}=310 \mathrm{kA}\right), B_{t}=$ $1.9 \mathrm{~T}$, the DED current is ramped up between $1.5 \mathrm{~s}$ and $3 \mathrm{~s}$ and is then kept constant to $3.5 \mathrm{~s}$, cocurrent neutral beam heating (NBI-Co $=1.5 \mathrm{MW}$ ) is applied, and the plasma is shifted by $5 \mathrm{~cm}$ to the high field side $(R=1.7 \mathrm{~m}, a=$ $0.42 \mathrm{~m}$ ); the resulting edge safety factor is $q_{a}=3.5$. In this example, the DED current is slowly ramped up to the full current of $7.5 \mathrm{kA}$ per coil [Fig. 2(b)] . One observes that the density increases with DED current, however, not linearly but stepwise [Fig. 2(a)]. The first step starts at a DED current of about $3.6 \mathrm{kA}$, and the next step at a current of about $6 \mathrm{kA}$. In between the steps, the density is nearly constant. The density trace in reference discharges without DED remain constant in time. A boronization was instrumental for the confinement improvement because in cases

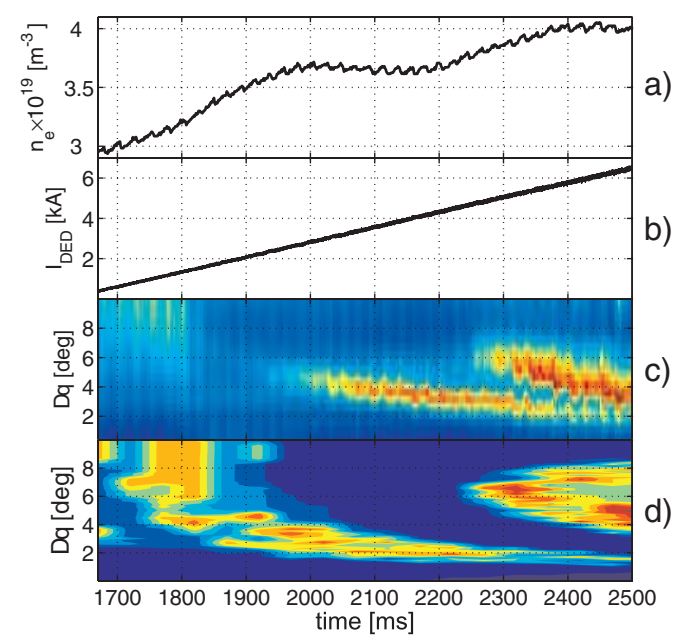

FIG. 2 (color online). Time traces of density (a) the DED current (b), time trace of the thermographic line scan in the time interval $1.7 \mathrm{~s} \leq t \leq 2.5 \mathrm{~s}$ (c), and calculated connection lengths of magnetic field lines hitting the target plate (d); the connection length is decoded by color. New substructures of the heat flux pattern appear when flux tubes starting at the unstable $X$ point of an island chain opens toward the wall. late after the wall conditioning the plasma develops easily tearing modes at enhanced DED currents; therefore, the disruption-free operation is limited to a DED current of about 4-5 kA. Density increase with ergodic field has been observed previously, but not the stepwise increase. These previous results were also obtained in high field side limited plasmas but with lower hybrid rather than neutral beam heating and with $q a=3.0$ [4].

At the onset of the steps, one observes rather abrupt changes in the heat deposition pattern as shown in Fig. 2(c). The line scans are oriented poloidally, i.e., perpendicular to the direction of the divertor strips and the development of two DED stripes is shown. With the onset of a step in the electron density, a new substructure starts which moves to the edge of the pattern until a new pattern starts. The old pattern then fades away and becomes less clear without actually disappearing. The structures have been understood as magnetic flux tubes which successively connect deeper island chains with the wall. The resulting theoretical structure is depicted in Fig. 2(d) showing the connection length of the magnetic field lines hitting the target plate. Since the stepwise density increases are generally connected with the new substructures, we assume that both phenomena have a common cause.

The development of the electron density is seen in Fig. 3. At the start of the DED phase the maximum density amounts to $2.5 \times 10^{19} \mathrm{~m}^{-3}$; in reference discharges without DED this density is maintained during the discharge. With DED, the density at the plasma edge remains unchanged but peaks in the plasma core reaching a value of (a)

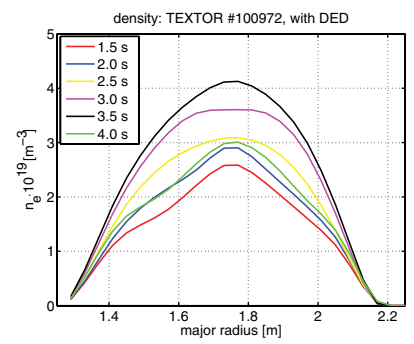

(c)

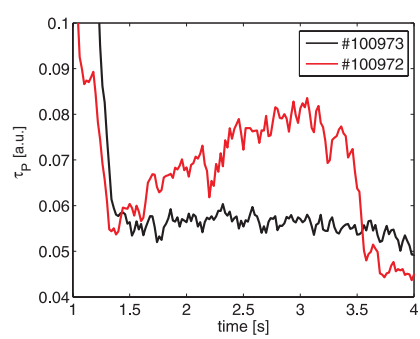

(b)

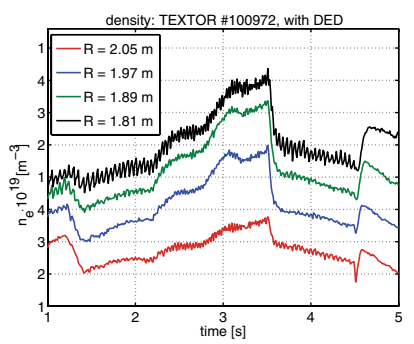

(d)

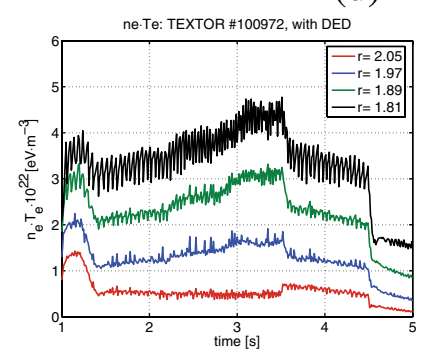

FIG. 3 (color online). Development of the radial plasma density profile (a); time traces the density (b), of particle confinement time of the discharge with DED (red) and a reference discharge without DED (black) (c), and of the electron pressure (d). 
about $4.5 \times 10^{19} \mathrm{~m}^{-3}$. After the DED phase, the initial density profile before the DED phase is restored again. Figures 3(b)-3(d) are time traces of the electron density, of particle confinement time of the discharge with DED (red) and a reference discharge without DED (black), and the electron pressure for the outer half of the discharge, respectively. One clearly sees the stepwise increase of the electron density as it was already discussed in Fig. 2. The density increase is connected with an increase of $\tau_{p}$ and a decrease of the particle diffusion coefficient. The increase of the electron density leads to a decrease in the electron temperature; due to edge ergodization, the decrease is stronger at the plasma edge and less pronounced in the core. The product of electron density and temperature increases over most of the profile with exception of the very plasma edge. This drop of the edge plasma temperature may be favorable for the plasma wall interaction. However, one has to keep in mind that the edge plasma properties are highly three dimensional, and that the plasma energy is transported along thin flux tubes from inner regions to the wall, despite lower average temperature does not exclude local peaks of plasma temperature at the walls. The improved confinement is primarily due to a spontaneous density increase for the time when the DED field is switched on. The same global confinement time is reached when the density is increased by gas puffing. However, the density increase is only observed at selected conditions of the edge safety factor and DED amplitude and decays again after the DED switch-off while the density after gas puffing remains.

The ion features of the plasma and the toroidal rotation are measured by charge exchange recombination spectroscopy (CXRS) on carbon impurities. At the very edge of the plasma one observes a decrease of the ion temperature, probably due to ergodization, and further inside the ion temperature. The changeover zone from reduced ion temperature to enhanced one is close to the $q=2$ surface. The toroidal rotation of the plasma grows over the whole profile with the highest increase in the gradient zone of the plasma. A similar plasma rotation in codirection due to the DED has been reported previously [12].

Below we discuss a possible mechanism of the observed improved confinement regime of the plasma during the DED operation. One expects that the DED perturbation field creates a specific magnetic structure with a transport barrier separating inner region with a low transport and outer region with the enhanced transport rate due to open chaotic magnetic field lines to the plasma wall. The structure of chaotic magnetic field lines are studied using a model developed in $[9,13]$ which uses Hamiltonian formulation of the field line equations assuming linear superposition of the plasma equilibrium field and the resonant magnetic perturbations in vacuum approximation. Hamitonian equations are integrated using a fast running symplectic mapping [14].
Resonant magnetic perturbations created by the DED destroy rational magnetic surfaces with $q=m / n$ forming $m: n$ magnetic islands at the plasma edge. Interactions of these islands give rise to chaotic field lines at the edge region of the plasma. Typically, the chaotic (ergodic) region has a finite radial extent restricted between the last intact (KAM) magnetic surface and the plasma wall. This system should be considered as an open chaotic system since field lines (actually, particles predominantly following them) are terminated after hitting the plasma wall $[9,15]$.

The typical structure of such a system is shown by the Poincaré section and the so-called laminar plot of field lines in Figs. 4 (a) Poincaré section, (b) laminar plot of field lines at the DED current of $1.35 \mathrm{kA}$, (c) Poincaré section, and (d) laminar plot of field lines at the DED current of $6.3 \mathrm{kA}$ for the plasma parameters corresponding to the discharge in Fig. 2. The chosen DED currents correspond to the first step and the third step of the density increase. The laminar plot is a contour plot of wall to wall connection lengths (in poloidal turns) of field lines $[15,16]$. The figures clearly show that at the onset of the first density step the island chain with the mode numbers $m: n=5: 2$ and that at the third step the island chain $m: n=4: 2$ starts to be ergodized at the unstable $X$ points. At the intermediate step one observes the ergodization of the secondary island chain $m: n=9: 4$. The corresponding $q$ surfaces are $q=2.5,2.25$, and 2 .

From the heat flux measurements which are in agreement with the theory [17] we conclude that the open ergodic structures develop a short connection length of
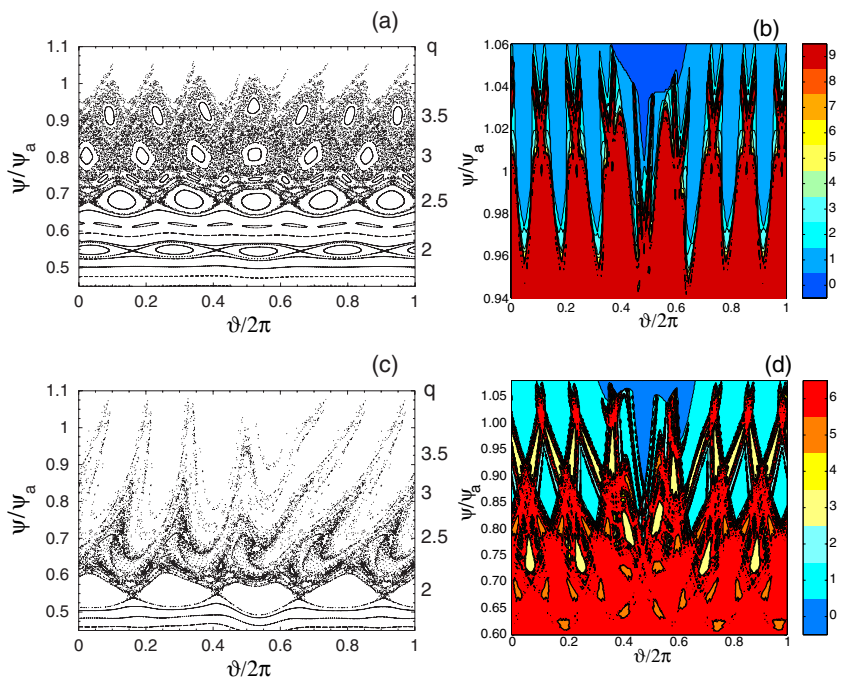

FIG. 4 (color online). (a) Poincaré section, (b) laminar plot of field lines at the DED current of $1.35 \mathrm{kA}$, (c) Poincaré section, (d) laminar plot of field lines at the DED current of $6.3 \mathrm{kA}$ for the plasma parameters corresponding to the discharge in Fig. 2. The chosen DED currents correspond to the first step and the third step of the density increase. For the plots intrinsic coordinates are used such that the Shafranov shift does not appear. 
magnetic field lines which carry a substantial part of the plasma flux [18]. These short connection length flux tubes should also transport efficiently the electric potential from the walls deep into the plasma, namely, to the deepest perturbed island chains. This statement is in agreement with a previous observations on TEXT [19] where the potential was measured by a heavy ion probe; the electric field penetrates roughly to the $q=2$ surface and influences the diffusion coefficient. Probe measurements on CSTN-II [20] show as well the modification of the electric field in the ergodic region.

In summary, we have found discharge conditions with a high degree of ergodization on the plasma edge and which form density barriers near the ergodic zone. The plasma energy loss in the ergodic zone is overcompensated inside the barrier region such that the total energy content of the plasma is increased by up to $20 \%$. The barrier formation occurs stepwise with increasing DED current. The step formation is linked with substructures of the heat deposition pattern; this feature has a generic character. The substructures have been identified as the result of magnetic flux tubes which connect the periodic fixed points of the magnetic islands. Along the flux tubes, the plasma energy is carried outwards to the wall, and forms a short circuit for the electric potential between the wall and the island chains. In the laminar plot, in particular, of Fig. 4(d), one observes that magnetic flux tubes with a total connection length of 5 poloidal turns at full DED current reach close to the $q=2$ surface, which means that the path from the $q=$ 2 surface to the wall is at most 2.5 poloidal turns long. This supports the hypotheses that the potential change deep inside the plasma is the cause for the observed density barrier formation where the islands separate regions of low and high transport [21], to which the magnetic field ergodization is most effective. Consistently we observe the highest improvement at a low density and a well heated plasma of low collissionality. The general assumption of the stability of a tokamak discharge is that the $q=2$ surface should remain - nearly - intact. We find the optimum conditions when the tangles to the island chain at the $q=2$ island is just starting to open.

This work was partially supported by Deutsche Forschungsgemeinschaft through Sonderforschungsbereich No. 591 and by the European Communities under the contract of Association between EURATOM/FZJülich, carried out within the framework of the European Fusion Development Agreement.

[1] P. Ghendrih, M. Becoulet, L. Colas, A. Grosman, R. Guirlet, J. Gunn, T. Loarer, A. Azéroual, V. Basiuk, and B. Beaumont et al., Nucl. Fusion 42, 1221 (2002).
[2] H. Tamai et al., Proceedings of the 15th International Conference on Plasma Physics and Controlled Nuclear Fusion Research, Seville, Spain, October, 1994 (IAEA, Trieste, 1995), Vol. 1, p. 137.

[3] A. Grosman, J.-M. Ané, P. Barabaschi, K.H. Finken, A. Mahdavi, P. Ghendrih, G. Huysmans, M. Lipa, P. R. Thomas, and E. Tsitrone, J. Nucl. Mater. 313-316, 1314 (2003).

[4] T. E. Evans, M. Gorniche, and A. Grosman et al., J. Nucl. Mater. 196 \& 198, 421 (1992).

[5] T. C. Hender, R. Fitzpatrick, A. W. Morris, P. G. Carolan, R. D. Durst, T. Edlington, J. Ferreira, S. J. Fieliding, P. S. Haynes, and J. Hugill et al., Nucl. Fusion 32, 2091 (1992).

[6] R. Fitzpatrick, Nucl. Fusion 33, 1049 (1993).

[7] T.E. Evans, R. A. Moyer, P. R. Thomas, J. G. Watkins, T.H. Osborne, J.A. Boedo, E. J. Doyle, M.E. Fenstermacher, K. H. Finken, and R. J. Groebner et al., Phys. Rev. Lett. 92, 235003 (2004).

[8] K. H. Finken, Fusion Eng. Des. 37, 335 (1997).

[9] K. H. Finken, S. S. Abdullaev, M. Jakubowski, M. Lehnen, A. Nicolai, and K.H. Spatschek, The Structure of Magnetic Field in the TEXTOR-DED, Energy Technology Vol. 45 (Forschungszentrum Julich, Julich, Germany, 2005).

[10] K. H. Finken, S. S. Abdullaev, A. Kaleck, and G. H. Wolf, Nucl. Fusion 39, 637 (1999).

[11] M. W. Jakubowski, S. S. Abdullaev, K. H. Finken, and the TEXTOR Team, Nucl. Fusion 44, S1 (2004).

[12] K. H. Finken, S.S. Abdullaev, M. F. M. deBock, M. von Hellermann, M. Jakubowski, R. Jaspers, H. R. Koslowski, A. Krämer-Flecken, M. Lehnen, and Y. Liang et al., Phys. Rev. Lett. 94, 015003 (2005).

[13] S. S. Abdullaev, K. H. Finken, and K. H. Spatschek, Phys. Plasmas 6, 153 (1999).

[14] S.S. Abdullaev, Construction of Mappings for Hamiltonian Systems and Their Applications, Lecture Notes in Physics Vol. 691 (Springer-Verlag, Berlin, 2006).

[15] S. S. Abdullaev, T. Eich, and K. H. Finken, Phys. Plasmas 8, 2739 (2001).

[16] T. Eich, D. Reiser, and K. H. Finken, Nucl. Fusion 40, 1757 (2000).

[17] T. E. Evans, R. K. Roeder, J. A. Carter, and B. I. Rapoport, Contrib. Plasma Phys. 44, 235 (2004).

[18] M.W. Jakubowski, O. Schmitz, S.S. Abdullaev, S. Brezinsek, K.H. Finken, A. Krämer-Flecken, M. Lehnen, U. Samm, K.H. Spatschek, and B. Unterberg et al., Phys. Rev. Lett. 96, 035004 (2006).

[19] S. C. McCool, A. J. Wootton, M. Kotschenreuther, A. Y. Aydemir, R. V. Bravenec, J.S. De Grassie, T.E. Evans, R. L. Hickok, B. Richards, and W. L. Rowan et al., Nucl. Fusion 30, 167 (1990).

[20] S. Takamura, H. Yamada, and T. Okuda, Nucl. Fusion 28, 183 (1988).

[21] R. Balescu, Phys. Rev. E 58, 3781 (1998). 